\title{
Ways to improve safety in the power industry: an automated hardware complex for pre-shift inspection of personnel of power enterprises
}

\author{
Robert Hizbullin* and Radik Hizbullin \\ Institute of Electric Power Engineering and Electronics, Kazan State Power Engineering University, 51 Krasnoselskaya Street, Kazan \\ 420066, Russian Federation
}

\begin{abstract}
This article discusses the possibility of using an automated medical hardware complex at energy enterprises that provides monitoring of the state of health of enterprise personnel. The complex under consideration includes individual functional devices with technical characteristics that ensure, during a preshift inspection, high reliability of the registered physiological parameters of the examined workers and, as a result, the reliability of monitoring the state of health of the examined.
\end{abstract}

\section{Introduction}

Works associated with the operation and repair of energy facilities and equipment can be hazardous to the health of personnel. Personnel with certain access to sensitive and dangerous energy facilities as well as electrical and high-altitude work may be subject to various psychophysical effects that affect the general physical state of the employee during a work shift.

In the Russian Federation, there are industry documents regulating measures to ensure the reliability of production activities and preserve the health of workers "Regulation on the psychophysical ensuring the reliability of professional activities and maintaining the health of personnel of energy enterprises" RAO UES dated 18/06/2000. [1]. This document defines the organizational and legal foundations of psychological support for the reliability of professional activities and the preservation of staff health. Tasks of psychophysical support are realized according to the results of surveys, including the process of pre-shift examination of personnel.

In addition to the above Regulations, there is Order of the Ministry of Energy of the Russian Federation of August,31 2011 N 390 "On approval of the Procedure for conducting medical examinations (surveys) of employees directly engaged in work related to servicing electrical power facilities" [2], in accordance with part 3 of Article 28 of the Federal Law of March, 6, $2003 \mathrm{~N}$ 35 "On power industry" [3], that determines the procedure for conducting medical examinations (surveys) of employees directly engaged in work related to the maintenance of energy objects. In accordance with this Order, at the request of the employer, pre-shift medical examinations can be carried out to establish the fact of the use of alcohol, drugs or psychotropic substances and psychophysical state of personnel where there are mandatory points for measuring blood pressure, heart pulse, body temperature.

A similar Order exists in the Ministry of Health and Social Development of Russia 12/04/2011 № 302n “On approval of the lists of harmful and (or) hazardous production factors and work during which the preliminary and periodic medical examinations are carried out, and the Procedure for conducting mandatory preliminary and periodic medical examinations (surveys) of employees engaged in hard work and work with harmful and (or) dangerous working conditions" [4].

In the Order of the Ministry of Health of Russia dated November,8, 2012 "On approval of the procedure for pre-shift (pre-trip) and post-shift ( post-trip) medical examinations" [5] in accordance with it.7 article of the Federal law dated 21/11/2011 № 323 "On the basics of protecting public health in Russian Federation" [6], there is a procedure of pre-shift (pre-trip) medical examinations, the first paragraph of which indicates: "Pre-shift (pre-trip) medical inspections are carried out before start of the work shift (trip) in order to identify signs of exposure to harmful and dangerous production factors, conditions and diseases that impede the performance of labor duties, including alcohol, drug or other intoxication and residual effects of such intoxication".

To fulfill the requirements of the above orders, medical and technical support is necessary to carry out pre-shift examination no more than 30 minutes before the start of the work shift $[3,4]$. Thus, it is necessary to determine quickly and accurately the health status of a certain number of personnel in a short period of time.

* Corresponding author: Robert.khizbullin@ mail.ru 


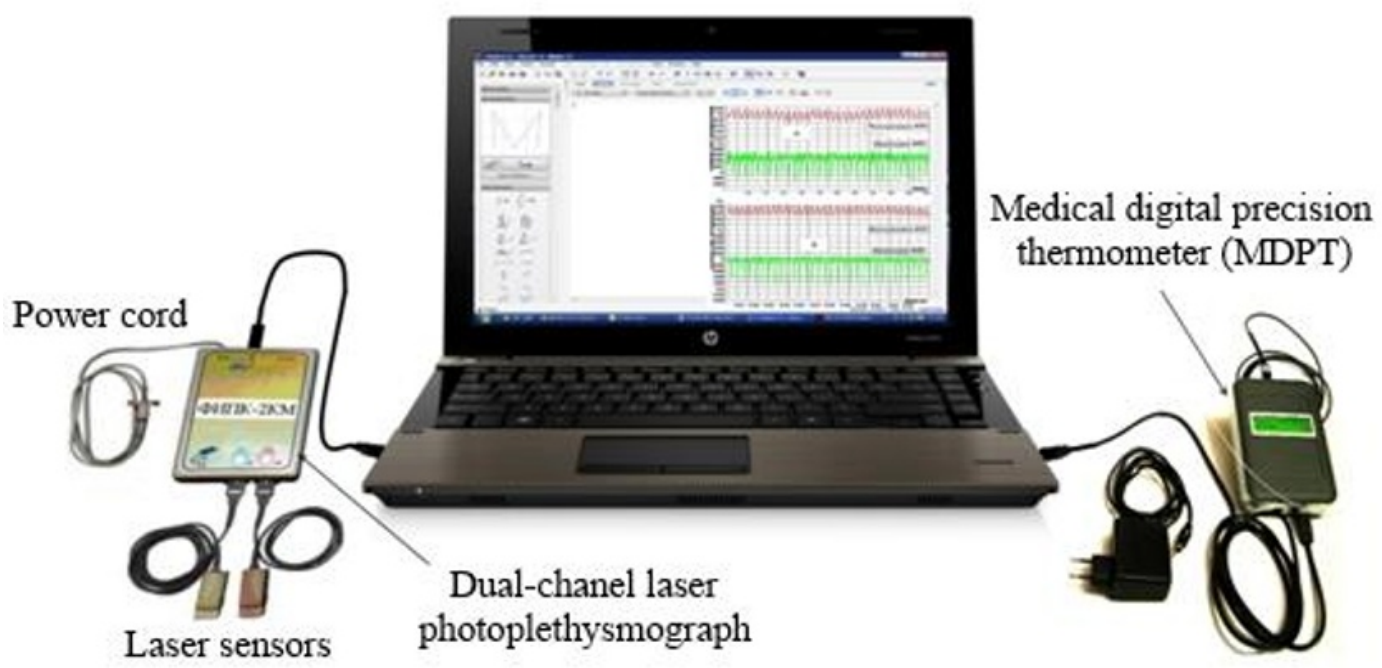

Fig. 1. Computerized hardware complex.

\section{Solution of the actual problem and practical implementation}

An automated medical complex for pre-shift examination of workers at energy enterprises has been developed to help doctors who monitor the physical condition of persons allowed to work. This computerized hardware complex (Figure 1) consists of the following functional parts: a medical precision thermometer, twochannel photoplethysmograph, and in the future it will be equipped with a three-channel pulse-oximeter.

The main task of the developed automated medical complex for pre-shift examination (survey) is to identify with high reliability the psychophysical state of the employee as well as the predictability of the his state for the period of the work shift. Also the medical worker must quickly and accurately make a decision on the admission of the employee to work and an additional post-shift inspection of the employee. All data at each examination are recorded in a database of pre-shift examinations stored in electronic form.

As already noted above in the regulatory documents, the mandatory procedures during the examination are taking readings of temperature, blood pressure, heart rate. In the developed automated complex temperature, pressure, heart rate measurement can be carried out at the same time, which reduces the examination time.

In medicine, temperature is an important physiological parameter, closely related to the physiological processes taking place in the body. Therefore, temperature measurement in medical diagnostics is a mandatory procedure.

The temperature range of measurement of the developed medical digital precision thermometer (MDPT) is: $+5 \ldots+50^{\circ} \mathrm{C}$, accuracy is within $0.05 \ldots$ $0.1{ }^{\circ}$ C. For a high-quality visual representation of temperature measurements, and relative measurements, as well as for graphical display of temperature changes, it is important to have high linearity and resolution of the measuring technical system. The temperature resolution should not exceed $0.01^{\circ} \mathrm{C}$.

Only a low-inertia system can provide high-quality registration of the temperature changes in the body, i.e. one whose inertness is at least an order of magnitude less than the temperature inertness of the measured area of the body. It follows that the temperature sensor should have the smallest overall dimensions and thermal inertia.

As sensors that allow recording the temperature with an accuracy of $0.1^{\circ} \mathrm{C}$ and having the smallest overall dimensions at the highest temperature sensitivity in the range of $+5 \ldots+50^{\circ} \mathrm{C}$, thermistors are most widely used in medicine [7].

The general technical requirements for medical thermometers [8-12] are fully met by the digital precision thermometer described in [13]:

-temperature range $+5 \ldots+50{ }^{\circ} \mathrm{C}$;

-relative error of the device when measuring temperature of $0.1 \%$;

-thermistor as a temperature sensor;

-absolute error of temperature measurement with a non-calibrated thermistor in the range of $+25 \ldots+50^{\circ} \mathrm{C}$ is $0.1^{\circ} \mathrm{C}$ (additional calibration is possible);

-measurement pace -15 values per second.

The developed medical digital precision thermometer (MDPT) (Figure 2), included in the automated complex consists of two main parts: a temperature recording unit and a temperature sensor having a remote sensing element (temperature sensor) - thermistor. This sensor allows you to record the temperature with an accuracy of $\pm 0.1^{\circ} \mathrm{C}$.

The length of the active part of the sensor is $450 \mathrm{~mm}$, diameter is $1 \mathrm{~mm}$, diameter of the thermistor head is 1.3 $\mathrm{mm}$. The sensor is connected to the unit using a $6.35 \mathrm{~mm}$ JACK connector. The total length of the sensor, including the supply cable and connector, is $1.5 \mathrm{~m}$. The 
sensor can be sterilized using any hospital disinfectant, such as ethanol, isopropyl or chlorite compounds.

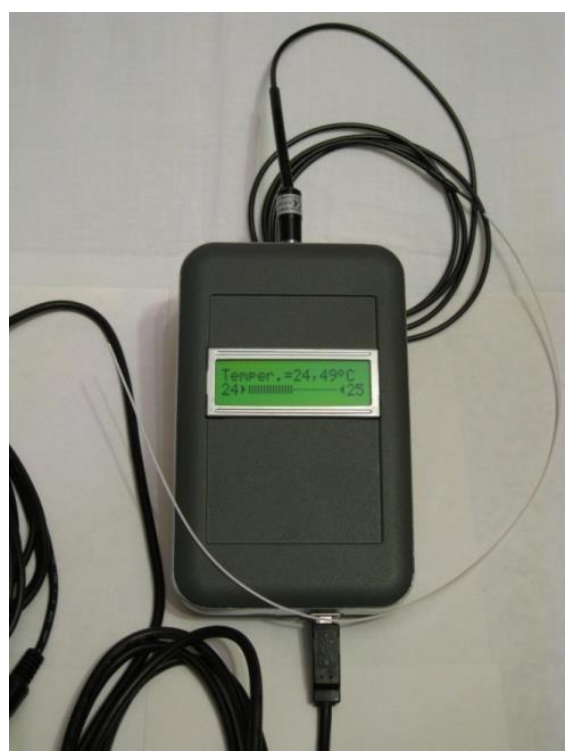

Fig. 2. Medical digital precision thermometer (MDPT).

The registration unit processes the sensor signal and displays it on the screen. As a power source of the main unit, either USB 2.0 interface or an external medical power source (a special adapter with a constant voltage in the range $+7.5 \ldots+12 \mathrm{~V})$ can be used. When connecting the unit via USB 2.0, temperature data transmitted on computer and then displayed through specialized software, stored in the computer's memory in the form of a simple text file.

The structural diagram of the thermometer is shown in figure 3 [14]. The temperature sensor signal goes the amplifier, then to an analog-to-digital converter (ADC), where it is digitized and converted into a digital code, which through galvanic isolation will be transmitted to the microcontroller. Microcontroller processes the received data, calculates the temperature value, displays received information on the display and transmits temperature data in digital code through a galvanically isolated USB 2.0 interface to a computer. The circuit includes a power selector.

The second device of the automated complex is a dual-channel photoplethysmograph, which operates on the basis of the finger photoplethysmography method.

The use of finger photoplethysmography is of great diagnostic value in the quick and accurate assessment of local capillary blood flow, measurement of arterial and venous pressure, volume pulse, blood flow velocity, vascular tone, heart rate, minute and systolic blood volume. One of the features of the diagnostic capabilities of photoplethysmography is the ability to prevent negative reactions that arose as the result of exposure to physical factors at some energy production facilities (for example, nuclear energy, etc.), as well as identification of stressful conditions. The fact that digital photoplethysmography for a short time provides accurate and objective information about changes in blood circulation parameters under various physical factors makes it possible to effectively use it in assessing the physiological state of technical staff and workers at energy production facilities, where pathogenic factors affect the human body. The diagnostic capabilities of photoplethysmography allow us to predict the permissible dose of pathogenic physical exposure factor and prevent negative reactions as a result of an overdose of this pathogenic factor affecting the body.

The photoplethysmographic signal is associated with the hemodynamics of the whole organism, as it reflects the appearance of a pulse wave, and the connection with

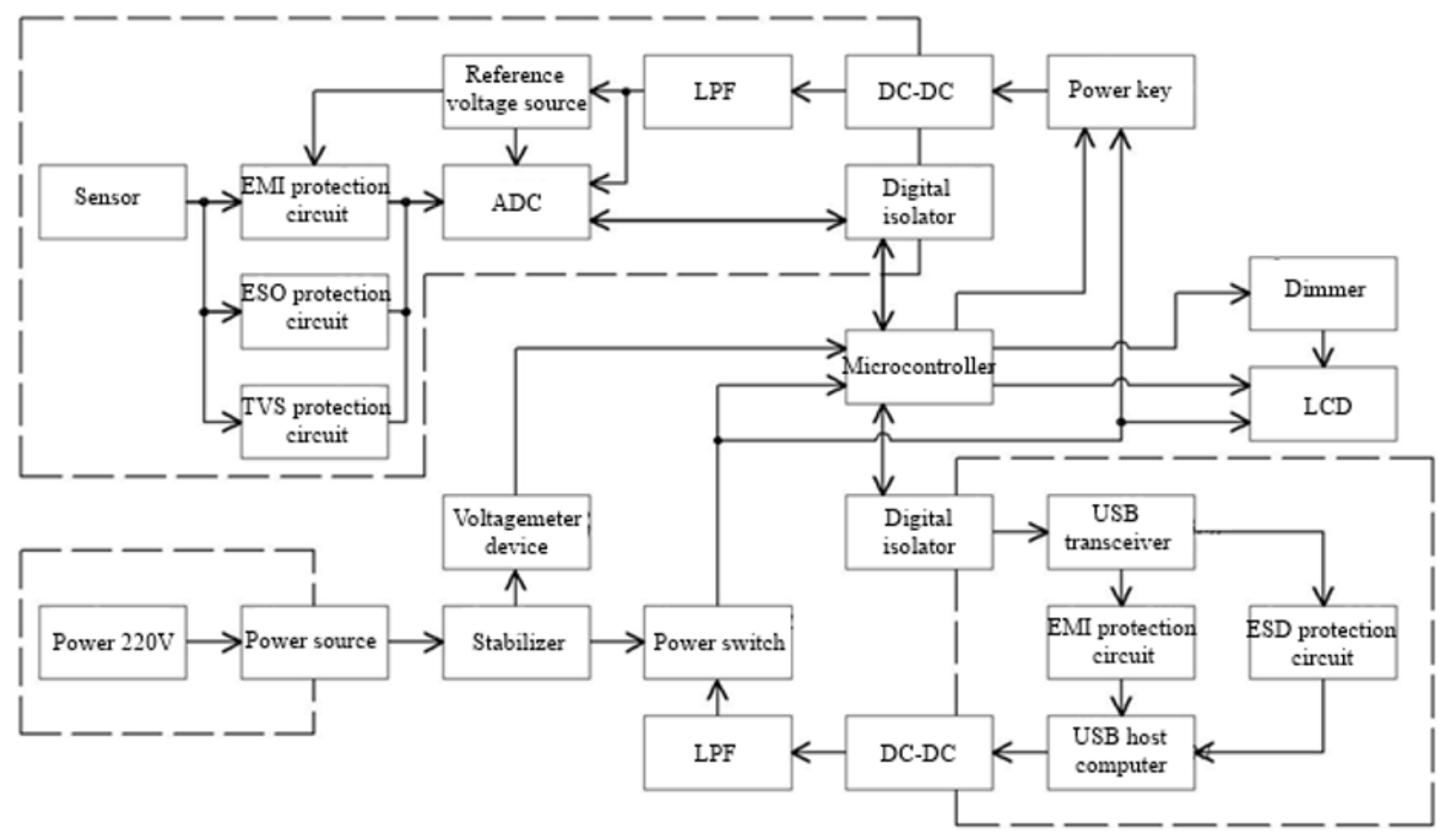

Fig. 3. The block diagram of thermometer (MDPT). 
hemodynamics is not direct, i.e. photoplethysmographic signal is the result of a "low- pass filter" on the pulse wave. From this it follows that the analysis of the shape of the variable component of the signal can provide information about both local blood circulation and the hemodynamics of the body as a whole. That is why photoplethysmographs recently are increasingly used in medical diagnostics.

In addition to the continuous recording of the photoplethysmogram, an occlusion test is possible as part of the examination. At the time of the development of the two-channel photoplethysmograph, there were publications on the use of single-channel finger LED $(\lambda$ । $940 \pm 20 \mathrm{~nm}$ ) photoplethysmography for medical purposes. The use of a single photosensor (one channel) with a radiation wavelength of $920 \ldots 960 \mathrm{~nm}$ will inevitably lead to "physiological interference" when registering a FPG signal due to different amounts of light absorption by the main informative components of blood flow - oxy- and deoxyhemoglobin. Therefore, it is impossible to obtain acceptable accuracy, repeatability and adequacy of PPG signal. Therefore, a new laser $(\lambda=$ $805 \pm 0.75 \mathrm{~nm}, \mathrm{P}=0.1 \ldots 0.2 \mathrm{~mW}$ ) dual- channel dualfinger photoplethysmograph was developed. The radiation wavelength $804.25 \ldots 805.75 \mathrm{~nm}$ is the "isobetic point" for oxy and deoxyhemoglobin, that is, the wavelength at which the spectral characteristics of these two substances coincide. So "physiological interference" can be avoided when receiving a photoplethysmogram.

In the previously developed photoplethysmographs the individual physiological state of the examined individuals was ignored when exposed to an external "energy" load. Physical factors such as electromagnetic radiation, radioactive radiation, electrical, magnetic, mechanical, and other factors are susceptible to the human body and seriously affect the human physiological state. Therefore, it is important to take into account the individual physiology of the worker, because the photoplethysmograms of different people may have strong variations in the value of the constants, connected with different optical densities of human tissues. The developed two-channel photoplethysmograph takes into account individual physiological state of the employee being examined, therefore the doctor receives a photoplethysmogram, which reflects reliable information about the worker. The device also allows artificial occlusion through an ordinary cuff. For this, the device has a valve and a compressor with a pressure sensor. Occlusive photoplethysmography is widely used in the diagnosis of vascular tone.

The main technical characteristics of the device PPG2KL [15]:

-registration, display and on-line linear filtering of biomedical information;

-support for standard (Nell-Cor-compatible) and laser photoplethysmographic sensors;

-synchronic registration of photoplethysmographic signal and pressure;

-the use of laser diodes for the operationof the device at isosbestic point $(805 \mathrm{~nm})$; -wide dynamic range (photocurrent conversion coefficient varies in the range of $2 \quad 105 \ldots 2 \mid 106$ ), as a result the device can be used for studies of peripheral blood flow;

-two- channel operation mode. Both channels operate in parallel with a sampling frequency of $350 \mathrm{~Hz}$ per channel;

-useful signal band $0.1 \ldots 40 \mathrm{~Hz}$;

-automatic artificial occlusion using a compressor and electronic valve;

-option to control the level of artificial occlusion in accordance with the measurement procedure;

-software is supplied for the automated processing and analysis of the registered information is supplied.

The appearance of the device is shown in figure 4 (conditionally named PPG-2KL - dual- channel laser photoplethysmograph). .

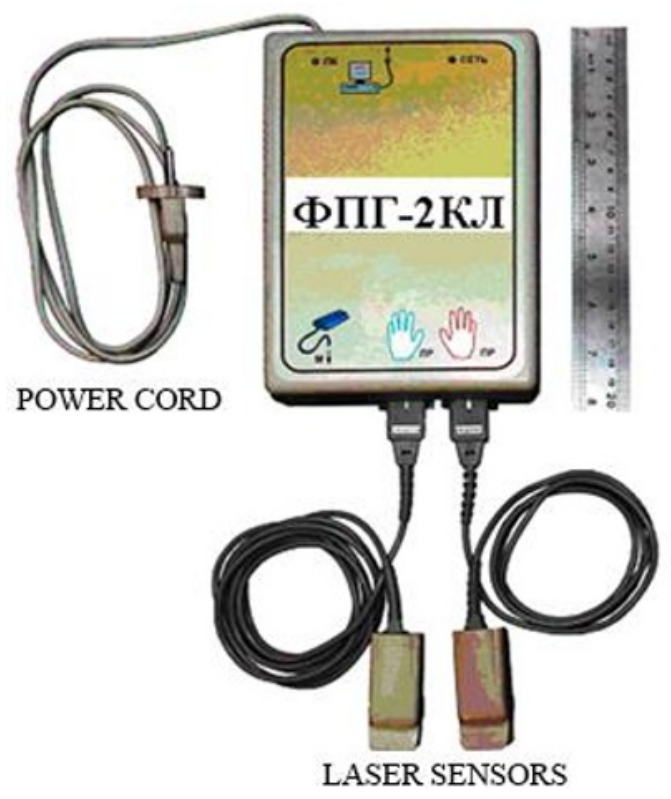

Fig. 4. Appearance of a dual- channel laser photoplethysmograph.

The block diagram of a dual- channel laser photoplethysmograph is shown in figure 5 .

\section{Conclusions}

The developed automated medical complex for pre-shift inspection (survey) of energy complex staff is an effective tool for medical workers. It allows to significantly reduce the time of pre-shift examinations (surveys) and to reliably diagnose deviations of physiological parameters of a person, such as temperature, pressure, pulse and other physiological parameters. The reliability of instrument readings proceed from the selected methodology and principles of instrument construction based on modern knowledge of the interaction of laser radiation with the main components of the blood flow.

A medical digital precision thermometer measures an important diagnostic parameter - the temperature of the employee being examined and together with the 


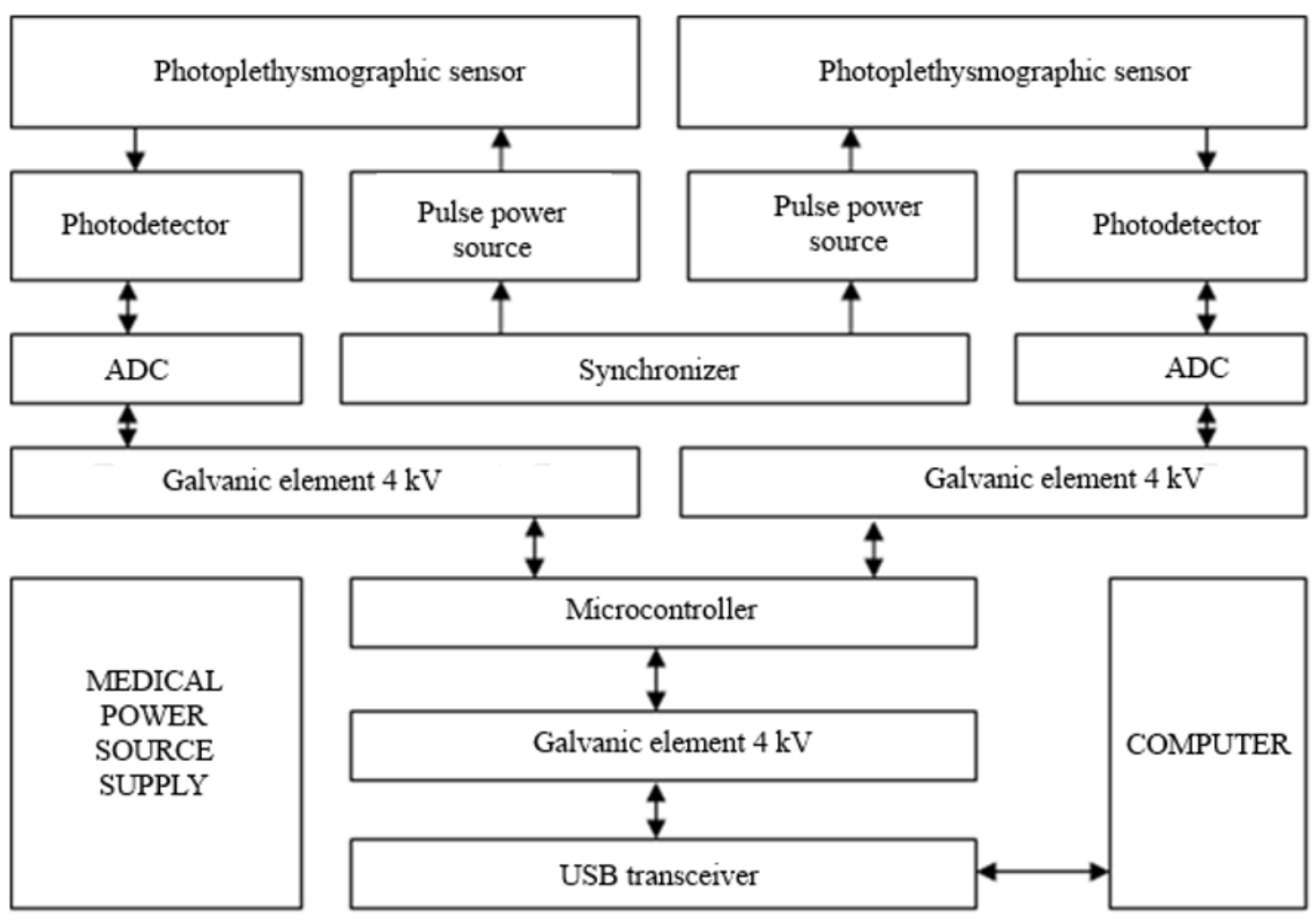

Fig. 5. Block diagram of a dual-channel laser photoplethysmograph.

photoplethysmogram it gives a more complete and accurate assessment of the physiological state of the employee.

A feature of the developed MDPT is temperature measurement with high accuracy and resolution of $0.01^{\circ}$ C. It ensures that even minor temperature measurements are recorded for diagnostic purposes and significantly expand the field of medical thermometry. It has automatic registration and recording of readings on a computer.

The advantages of the method of photoplethysmography in the developed photoplethysmograph PPG-2KL:

1. Provides registration of the reaction of the human body to an external physical effect of a pathogenic nature;

2. The use of a laser with a wavelength of $805 \mathrm{~nm}$ eliminates the possibility of "physiological interference" associated with a change in the ratio of hydroxy- and deoxyhemoglobin, as the spectral characteristics of the optical absorption of both types of hemoglobin intersect at an "isobestic point" at a wavelength of $805 \mathrm{~nm}$;

3. The use of a two-channel laser photoplethysmographic system for registration allows you to: compare both signals; improve the quality of the overall PPG signal; expand scope of photoplethysmographs.

4. The use of a two-channel laser photoplethysmograph as a recorder of changes in the state of the body that is affected by pathogenic external influences;
5. Photoplethysmogram in this aspect reflects the dynamics of the blood supply in the body. Registering such changes, we can evaluate the effects of external pathogenic effects and further to control the amount of such exposure in order to prevent negative consequences for the body as a whole.

\section{References}

1. Position of RAO "UES of RUSSIA" dated 06/06/2000, "Regulation on the psychophysical ensuring the reliability of professional activities and maintaining the health of personnel of energy enterprises", NRD 153-34.0-03.503-00 (2000)

2. Order of the Ministry of Energy of the Russian Federation of August 31, 2011, "On approval of the procedure for conducting medical examinations (surveys) of workers directly involved in work related to the maintenance of electric power facilities", 390 (2011)

3. Federal Law of March 26, 2003 N35-FZ "On Electric Power Industry", Collection of Legislation of the Russian Federation, 13, 1177(2003), 45, 5427(2007)

4. Order of the Ministry of Healthcare of Russia dated April 12, 2011, "On approval of the lists of harmful and (or) hazardous production factors and works during which preliminary and periodic medical examinations (surveys) are carried out, and the Procedure for conducting mandatory preliminary and periodic medical examinations (surveys) of 
employees engaged in heavy work and work with harmful and (or) dangerous working condition", 302n (2011)

5. Order of the Ministry of Health of Russia dated November 8, 2012, "On approval of the procedure for pre-shift (pre-trip) and post-shift (post-trip) medical examinations", (2012)

6. Federal Law of 21/11/2011 No. 323, "On the basis of the protection of public health in the Russian Federation", 323 (2011)

7. R.G. Jackson, Newest Sensors, Technosphere or Eds V.V. Luchinin, 133-138 (2007)

8. J.M. Zurbuchen, Precision thermometry, Measurement science conference tutorial "Thermometry-fundamental and practice", (2000)

9. B.H. Childs, Practical Temperature Measurement, Oxford, ISBN 0-750-65080-X (2001)

10. J.V. Nicholas, D.R. White, Traceable Temperatures, 2nd ed, ISBN 0-471-49291-4 (2001)

11. GOST R 50267.0.3-99. Medical electrical products

12. GOST R15.013-94. The system of development and production launch. Medical products

13. A.I. Larushin, M.A. Galkin, R.N. Hizbullin, V.A. Novikov, A.P. Kuzmich, Measurement of the temperature response of human organs to the electrolaser effect, World of Measurements, 3, 2125 (2010)

14. A.I. Larushin, M.A. Galkin, R.N. Hizbullin, V.A. Novikov, Medical digital precision thermometer, World measurements, 9, 9-17 (2010)

15. A.I. Larushin, M.A. Galkin, R.N. Hizbullin, V.A. Novikov, Dual-channel laser photoplethysmograph, World of measurements, 7, 22-28 (2010) 